ISSN 2090-3359 (Print)

ISSN 2090-3367 (Online)

\title{
$\mathbf{A} \mathbf{\Delta} \boldsymbol{\Sigma}$
}

\section{Advances in Decision Sciences}

\section{Volume 25 \\ Issue 2 \\ June 2021}

\author{
Michael McAleer \\ Editor-in-Chief \\ University Chair Professor \\ Asia University, Taiwan
}

Published by Asia University, Taiwan

ADS@ASIAUNIVERSITY 


\title{
Social Media and Analytics: A Case Study of Tweets Data *
}

\author{
Hak J. Kim ** \\ Department of Information Systems and Business Analytics \\ Hofstra University
}

Revised: May 2021

* The author thanks the reviewer for very helpful comments and suggestions.

** Correspondence: hak.j.kim@ hofstra.edu 


\begin{abstract}
Social media is emerging as a main communication channel in business and society. People use it in their daily life to share information with their friends and family. In this paper, we attempt to analyze Tweets data for understanding the relationship of social media and stock market as a case study. Data is collected more than 1.6 million tweets and RapidMiner software is used for their analysis. Our result shows that social media has relation with stock mark. It means that social media has an impact on financial market. However, there are some limitations to use social media for better decision making in investment. In a future paper, we need to develop more sophisticated analytics model and integrate different types of data to produce better results.
\end{abstract}

Keywords: Social Media, Big Data, Analytics, Twitter, Financial Stock Price.

JEL: C50, C90, C59, M15, M20. 


\section{Introduction}

Today's computing paradigm change is very much to some of the other shifts that have happened in information and communications technology (ICT) industry, such as mainframe to client/server and to cloud (Kuo, 2011). It is very different from previous shifts because of emerging multiple innovative technologies including mobile computing, cloud computing and social media technologies, . A bunch of different activities are happening whether it is social, whether it is mobility, whether it is cloud, and just as insatiable demand for information. It is like a huge storm or Tsunami [2].

As mobile cloud computing (Fernando et al., 2013) is taking over the position of the Internet, people can use their smartphones more often to communicate with family, friends, and other people regardless of time and place. Riche applications and faster services (i.e., 4G or 5G technologies) have brought explosive growth of social media market including YouTube, Twitter, Instagram, and Facebook.

We are certainly living in the era of social media. Social media today is popularized as new paradigm of communication. Unlike traditional structured data, social media data is unstructured like text and audio/video clips. In the past, those data could not be measured and analyzed because of lack of technologies and tools for collecting and analysing them.

Recent advancement of computing technologies and data science, the unstructured data can be collected and analysed. Social media data is a typical type of these unstructured data which is called 'Big Data'(Manyika et al., 2011). Social media analytics is simple a set of methodologies and techniques to collect and analyse social media data for producing meaningful information or insights.

Previous studies (Gruhl et al., 2005; Liu et al, 2007; Choi and Varian, 2009; Asur and Huberman, 2010) have been done related to social media. For instance, Karabulut (2011) analyzed Facebook's Gross National Happiness (GNH) index He concluded that GNH could predict daily returns in the US equity market. This paper builds upon keywords from tweets to create its own index rather than to use a published index. 
The paper presents to the understanding of social media analytics and applies it to the financial sector focused on a stock market as a case study. The purpose of this paper is to analyze social media data (that is, tweets) and identify its relationship with stock market data (that is, Dow stock price index). The assumption is that the keywords represent investor sentiment. This paper assumes that that an investor's sentiment plays an important role for making decision when they buy and sell stocks. As a result, tweets can affect the daily traction in the stock market. In the future, the analytics model will be built to predict the stock movement using any social media data.

This paper is organized as follows. The second section is briefly presents research methods and overviews data. A simulation model using RapidMiner is explained in the third section. The fourth section discusses simulation results and its findings. Finally, a conclusion and future paper are presented in the fifth section.

\section{Research Methods and Data}

\subsection{Hypotheses}

The paper establishes the following hypotheses:

- Hypothesis 1: There exists a positive correlation between real return and estimated return.

- Hypothesis 2: There is high fluctuation in real return and estimated return in the earlier periods from 2009 (financial crisis), whereas there is low fluctuation in the later periods from 2009.

- Hypothesis 3: Seemingly good words (i.e., good, up, high, buy...) will be associated with high returns, whereas bad words (i.e., bad, down, low, sell... ) will be associated with low returns.

\subsection{Data Collection and Cleaning}

The paper is based on the assumption that tweets reveal an investor's sentiment and thus provide a basis for predicting financial market. We collected over one million tweets data from 2006 to 2011. The primary data was the text file which has 1,608,621 rows representing each individual tweet. The data includes date and time, twitter ID, and keywords. Figure 1 shows a sample of raw tweets data. 


\section{[Figure 1 here]}

The data is broken into three groups equally: 536,207 tweets per group). We process data cleaning to each group's data set. First, a tweet data which is missing a keyword is removed because it presents no value to the analysis. Second, the date and time is shortened to include only the date and removed time data because we calculate only daily return (not time basis). Twitter ID data is also deleted because it is irrelevant for our data analysis. After data cleaning, data size are decreased from 1,608,621 rows and 4 columns (fields) to 1,130,577 rows and 2 columns (Table 2).

A new column is created for adding 'Return' data. Return is calculated by using the following single period arithmetic formula:

$$
R=\frac{P_{1}-P_{2}}{P_{1}}
$$

where $P_{1}$ represents the adjusted close price of the previous day.

$P_{2}$ represents the adjusted close price of the present day.

The data for the daily adjusted close price are taken from the S\&P500 index in Yahoo! Finance (2021).

\subsection{Modelling and Simulation}

The tool for tweets data analysis is RapidMiner (2021). It is a GUI mode open-source system with more than 500 operators for data mining and data analysis. The main components of RapidMiner are operator, process, and repository. Operator is simply a task to be processed. Processor is connecting between operators. Repository is a kind of storage that retrieve and save data.

The first step is to convert the raw data into a format readable for RapidMiner. After the data preparation stage, the next step is to find the return associated with each keyword. For example, the keyword "good" may be associated with positive returns, whereas the keyword "bad" may be 
associated with negative returns. Then, the next step is to develop an estimated (hypothetical) return schedule based on the returns associated with each keyword. The final step is to analyze the relationship between the estimated return schedule and the real return schedule. The hypothesis is that the estimated return schedule and the real return schedule will have a strong positive correlation.

Figure 2 shows our research model for simulation. The first process is the "Keyword" process which is to produce return relevant keywords. The three CSV files were imported using three "Read CSV" operators. The "Append" operator combined all three sets of data into a single set of data. "Select Attributes" operator selected only the relevant data columns to be used for the analysis.

\section{[Figure 2 here]}

Since the relevant columns are "Keyword" and "Return(date)", the "Aggregate" operator grouped the keywords and computed the average of Return(date). The "Write Excel" operator enabled the final results to be recorded in an Excel file. Return (keyword) column is the return associated with each keyword. It also has a "frequency" column which shows how many times a word appears in tweets. Low frequency shows that the return associated with the keyword is unreliable because of small sample size. As a rule of thumb, words with a frequency of less than 100 were deleted for increased accuracy in average return. For creating a process that yields a hypothetical or estimated return schedule based on the keywords, we create files for input. As shown in Figure 3, a column "Return(date)" is added to the original file using the LOOKUP function.

[Figure 3 here]

The file has columns to represent the day's return. Since there are multiple rows with the same date, a process is needed to perform aggregation. The process "Value" is able to group the rows with the same date, and compute the average of all the returns in that group. It is very similar to the "Keyword" process, but the only difference is that the "Value" process groups by date, whereas the "Keyword" process groups by keyword. Returns with a low sample size had to be deleted. Therefore, as a rule of thumb, any dates with less than 200 rows are deleted to improve accuracy. As shown Figure 4, it contains columns that represent the estimated returns corresponding to each date. 


\section{[Figure 4 here]}

Another column, "Return(date)", is added As shown in Figure 5. The column "Return(date)" represents the real return of that day, whereas "Estimated Return" represents the estimated or hypothetical return. This allowed for a comparison between the estimated return and the real return.

\section{[Table 5 here]}

A simple process called "CM" was used to find out the correlation between the estimated return and the real return. As shown in Figure 6, the process has three operators: "Read Excel", "Select Attributes" and "Correlation Matrix". The "Read Excel" operator takes in the Excel file. Then, the "Select Attributes" operator enables the selection of attributes that are relevant to this process. Finally, the "Correlation Matrix" computes the correlation between all the selected attributes. Instead of writing the result in Excel format, this process shows the results within RapidMiner.

\section{[Figure 6 here]}

\section{Results Analysis}

The analysis revealed some interesting results in the process. However, the analysis did not result in a useful model that could predict the future stock market changes due to many reasons.

Hypothesis 1: The hypothesis is rejected. The correlation matrix revealed a weak positive correlation between the estimated return and real return. As shown in Figure 7, the correlation between EC and RC was $19.2 \%$.

[Figure 7 here] 
The reason behind the low correlation may be the huge chunk of data loss from dates November 2009 to January 2010. The cause could not be identified. However, there are some possible explanations. Switching the file formats back and forth may have caused this loss. Accidental deletion in the process of handling data manually could be a cause. Using many different processes instead of using a combined single process in RapidMiner may have caused data loss (Figure 8)

\section{[Figure 8 here]}

Hypothesis 2: The hypothesis is accepted. In early periods of the data, real return had high fluctuations in daily price changes. However, estimated return did not experience high fluctuations in the early periods. The rationale behind the anticipated high fluctuation is the financial crisis of 2007-2008. The available data ranges from October 2006 to March 2010. This includes the 2007-2008 financial crisis period where daily changes can be extreme. As shown in Figure 9, the graph shows that there are high fluctuations in daily changes in the earlier periods close to the crisis and low fluctuations in the later periods.

\section{[Figure 9 here]}

However, the graph in Figure 10 does not present high fluctuations in the earlier periods.

\section{[Figure 10 here]}

Hypothesis 3: The hypothesis is partially accepted. The hypothesis is that seemingly good words were associated with high returns, whereas bad words were associated with low returns.

In Figure 11, the word associated with the highest returns was "acquiring". Interestingly, "acquired" and "acquires" had the third highest return and the fifth highest return respectively. Also, "merge" and "merger" had the eighth and ninth highest return. It is unclear why words related to mergers and acquisitions are associated with high return in the stock market. Other words associated with high returns include: "higher", “upside”, “gain”, “up", "profit”..., and so on. The word associated with the 
lowest return was "downside". Words that are characterized by negative returns include: "down", "loss", "bottom", "low", "bad"..., and so on.

\section{[Figure 11 here]}

\section{Conclusion}

\subsection{Managerial Implications}

Meaning 1: We attempt to find the value of social media data through empirical data.

Meaning 2: Using the real social media data, we attempt to predict the future financial market.

\subsection{Limitations}

\section{Limitation 1: No previous studies}

There were many difficulties and problems associated with this analysis. The biggest difficulty was the availability of resources - the lack of previous studies on the subject forced the use of unproven methods and models. For instance, simple arithmetic mean was used when computing the returns associated with the keywords. Outliers may have had a big impact on skewing the arithmetic mean. Using other measures of central tendencies, such as median, may have helped increase the accuracy of the returns.

\section{Limitation 2: Reality Issue}

Data is not directly collected from the real-world. We got it from the vendor. There is an issue of reliability. It is impossible to get real data without the vendor support. 


\section{Limitation 3: Low performance system}

The analysis was done from a laptop computer with subpar processer and low RAM. RapidMiner would frequently lag and freeze when working with large amounts of data. Working in such conditions increased the time spent on importing data, converting format and producing results. The analysis would have gone much faster with a better computer, allowing additional analysis on the subject

In addition, through this opportunity, we were able to learn about RapidMiner and apply it in social media data. We saw much potential in RapidMiner as an analytics tool, and were constantly amazed by what it could accomplish. More analytical operators and functions are being added to RapidMiner, so that it presents opportunities for further research on this topic. 


\section{References}

Asur, S., and Huberman, B. (2010), Predicting the Future with Social Media arXiv:1003.5699v1

Choi, H \& Varian, H. (2009), Predicting the present with google trends, Technical report, Google.

Fernando, F., Loke, S., and Rahayu, W. (2013), Mobile cloud computing: A survey, Future Generation Computer Systems, 29(1), 84-106.

Gruhl, D, Guha, R, Kumar, R, Novak, J, \& Tomkins, A. (2005), The predictive power of online chatter, ACM, 78-87.

Kuo, C. (2011), Paradigm Shifts in Modern ICT Era and Future Trends, In Proceedings of the $10^{\text {th }}$ International Symposium on Signals, Circuits and Systems (ISSCS).

Liu, Y, Huang, X, An, A, \& Yu, X. (2007), ARSA: a sentiment-aware model for predicting sales performance using blogs, ACM, 607-614.

Manyika, G., Chui, J., Brown, M. Bughin, B., Doobs, J., Roxburgh, R., and Byers, A. (2011), Big Data: The Next Frontier for Innovation, Competition, and Productivity, Report, McKinsey Global Institute.

RapidMiner (2021), https://rapidminer.com/

Wegmuller, M., Weid, J., Oberson, P., and Gisin, N. (2000), High resolution fiber distributed measurements with coherent OFDR, in Proceedings ECOC'00, paper 11.3.4, 109.

Yahoo!Finance (2021) https://finance.yahoo.com/

Zhang, S., Zhu, C., Sin, J., and Mok, P. (1999), A novel ultrathin elevated channel low-temperature poly-Si TFT, IEEE Electron Device Lett., 20, 569-571. 


\section{Figure 1}

\section{Raw Tweets Data}

\begin{tabular}{|c|c|c|c|}
\hline 20070306212211 & $\$ \$ \$$ & \multicolumn{2}{|l|}{0005887328} \\
\hline 20070306221120 & $\$ \$$ & \multicolumn{2}{|l|}{0005888593} \\
\hline 20070307155450 & \$wife & 0005905967 & up \\
\hline 20070309014235 & $\$ \$$ & 0005947467 & bull, bought \\
\hline 20070309060022 & $\$$ ney & 0005959151 & soon \\
\hline 20070309152822 & $\$$ boss & 0006048741 & \\
\hline 20070309194559 & $\$$ boss & 0006122021 & today \\
\hline 20070312193229 & $\$ \ldots$ & 0007093051 & \\
\hline 20070313011634 & $\$ \ldots$ & 0007220691 & \\
\hline 20070313175359 & $\$ \$ \$$ & 0007466691 & \\
\hline 20070314220940 & $\$ \wedge$ & 0007944461 & \\
\hline 20070315042223 & $\$ k$ & 0008053671 & \\
\hline 20070316194743 & $\$ \$$ & 0008698881 & today, up \\
\hline 20070317025538 & $\$ \$$ & 0008823181 & bad \\
\hline 20070318024912 & $\$ \$ \$$ & 0009181191 & \\
\hline 20070318231503 & $\$ \$$ & 0009438481 & smart, buy, smart \\
\hline 20070319012838 & $\$$. & 0009479741 & year \\
\hline 20070319184415 & \multicolumn{3}{|c|}{ \$gruber_tweets 0009758071} \\
\hline 20070319184415 & \multicolumn{3}{|c|}{$\$ g r u b e r \_t w e e t s \quad 0009758071$} \\
\hline 20070320030941 & \$haun & 0009898901 & close \\
\hline 20070320065312 & $\$ p r o g$ & 0009958241 & \\
\hline 20070321185826 & $\$ \$$ & \multirow{2}{*}{$\begin{array}{l}0010625571 \\
0010868201\end{array}$} & \\
\hline 20070322045642 & $\$ k i n$ & & $\mid$ \\
\hline 20070322211343 & $\$ \$ \$ \$$ & 0011197911 & \\
\hline 20070323035817 & $\$ \$ \$$ & 0011359891 & update \\
\hline 20070323223522 & \$tonic. & 0011785361 & \\
\hline 20070323230843 & $\$ p r e f i x$ & 0011799091 & \\
\hline 20070324223325 & $\$ \$ \$ \$$ & 0012219241 & worth \\
\hline 20070325203510 & $\$$ food & 0012609141 & \\
\hline 20070325203510 & \multicolumn{2}{|c|}{ \$inboxSize } & 141 \\
\hline 20070325203510 & $\$$ sleep & 0012609141 & \\
\hline 20070325203510 & \$water & 0012609141 & \\
\hline 20070326003606 & $\$ \$$ & 0012710071 & \\
\hline 20070326132542 & \$hit & 0012972991 & hate \\
\hline 20070326132634 & \$hit... & 0012973531 & \\
\hline 20070326173024 & \$oft: & 0013084671 & made \\
\hline 20070327050826 & $\$$. & 0013446471 & \\
\hline 20070327215256 & $\$ \$ \$$ & 0013881081 & \\
\hline 20070327231511 & \$WORKSFO & DRME & 521 \\
\hline
\end{tabular}


Figure 2

A Simulation Model with RapidMiner

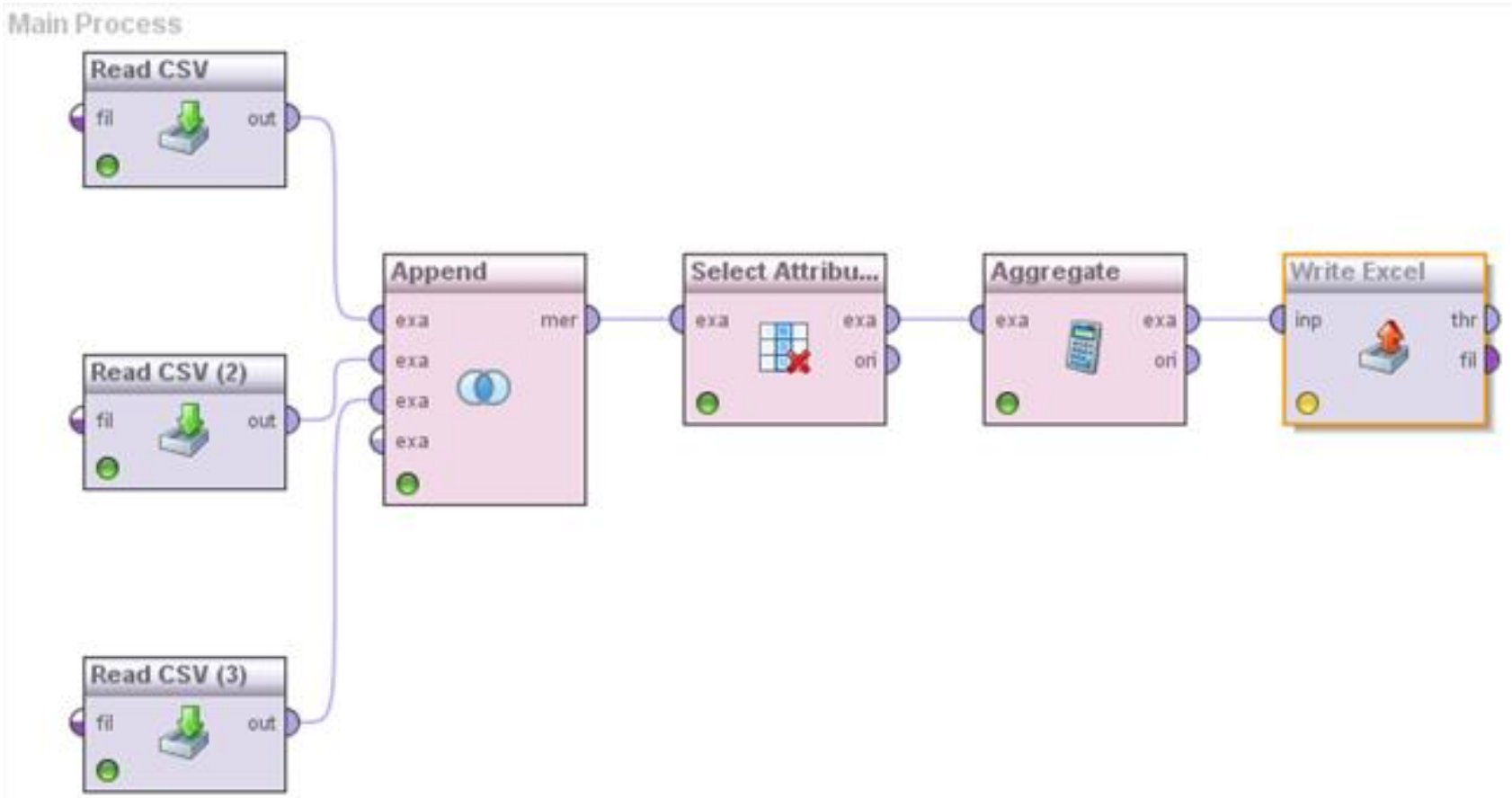


Figure 3

Result of Keyword Analysis

\begin{tabular}{l|r|r|}
\hline Keyword & Return(keyword) & Frequency \\
\hline acquire & 0.00131280 & 371 \\
\hline acquired & 0.00267631 & 293 \\
\hline acquires & 0.00255162 & 214 \\
\hline acquiring & 0.00319691 & 134 \\
\hline action & 0.00104051 & 3573 \\
\hline alpha & -0.00097745 & 645 \\
\hline announce & 0.00054675 & 667 \\
\hline announced & 0.00076710 & 1355 \\
\hline announcement & 0.00137009 & 619 \\
\hline announces & -0.00049764 & 945 \\
\hline announcing & -0.00064544 & 176 \\
\hline bad & -0.00006385 & 11646 \\
\hline bear & -0.00008253 & 2193 \\
\hline beta & 0.00168895 & 456 \\
\hline bond & 0.00036867 & 655 \\
\hline bottom & -0.00037005 & 2224 \\
\hline bought & 0.00075457 & 17442 \\
\hline bounce & -0.00052624 & 2186 \\
\hline breaking & 0.00057719 & 2180 \\
\hline breakout & 0.00266934 & 2846 \\
\hline bull & 0.00038834 & 3649 \\
\hline buy & 0.00060098 & 22565 \\
\hline buying & 0.00052332 & 7078 \\
\hline call & 0.00062581 & 11579 \\
\hline cap & 0.00023919 & 1351 \\
\hline cash & -0.00003897 & 6921 \\
\hline charges & 0.00089415 & 632 \\
\hline & & \\
\hline
\end{tabular}


Figure 4

\section{Estimated Return}

\begin{tabular}{|l|l|l|r|}
\hline \multicolumn{1}{|c|}{ A } & \multicolumn{1}{c|}{ B } & \multicolumn{1}{c}{ C } \\
\hline 1 & Date & Estimated Return & Keyword Per Day \\
\hline 2 & $09 / 15 / 2008$ & 0.0002798024093023 & 215.00 \\
\hline 3 & $10 / 06 / 2008$ & 0.0001025773080189 & 212.00 \\
\hline 4 & $10 / 10 / 2008$ & 0.0001732615737705 & 244.00 \\
\hline 5 & $10 / 16 / 2008$ & 0.0002552908557377 & 244.00 \\
\hline 6 & $10 / 17 / 2008$ & 0.0004193588322581 & 220.00 \\
\hline 7 & $10 / 21 / 2008$ & 0.0003545098779167 & 241.00 \\
\hline 8 & $10 / 22 / 2008$ & 0.0002512865661905 & 210.00 \\
\hline 9 & $10 / 23 / 2008$ & 0.0002895931437209 & 215.00 \\
\hline 10 & $10 / 24 / 2008$ & 0.0001388618072519 & 262.00 \\
\hline 11 & $10 / 27 / 2008$ & 0.0003592420009615 & 209.00 \\
\hline 12 & $10 / 28 / 2008$ & 0.0003757085487805 & 247.00 \\
\hline 13 & $10 / 29 / 2008$ & 0.0002786696621277 & 235.00 \\
\hline & & & \\
\hline
\end{tabular}


Figure 5

Return(date)

\begin{tabular}{|c|l|c|r|}
\hline \multicolumn{1}{|c|}{ A } & \multicolumn{1}{c|}{ B } & \multicolumn{1}{c|}{ C } \\
\hline 1 & Date & Estimated Return & Return(date) \\
\hline 2 & $09 / 15 / 2008$ & 0.0002798024093023 & -0.0471358951825517 \\
\hline 3 & $10 / 06 / 2008$ & 0.0001025773080189 & -0.0385178716010297 \\
\hline 4 & $10 / 10 / 2008$ & 0.0001732615737705 & -0.0117592755407068 \\
\hline 5 & $10 / 16 / 2008$ & 0.0002552908557377 & 0.0425074903066619 \\
\hline 6 & $10 / 17 / 2008$ & 0.0004193588322581 & -0.0062128208108365 \\
\hline 7 & $10 / 21 / 2008$ & 0.0003545098779167 & -0.0307996752587782 \\
\hline 8 & $10 / 22 / 2008$ & 0.0002512865661905 & -0.0610125124339040 \\
\hline 9 & $10 / 23 / 2008$ & 0.0002895931437209 & 0.0126340908584046 \\
\hline 10 & $10 / 24 / 2008$ & 0.0001388618072519 & -0.0345112376253978 \\
\hline 11 & $10 / 27 / 2008$ & 0.0003592420009615 & -0.0317643167535386 \\
\hline & & & \\
\hline
\end{tabular}


Figure 6

\section{Correlation Matrix}

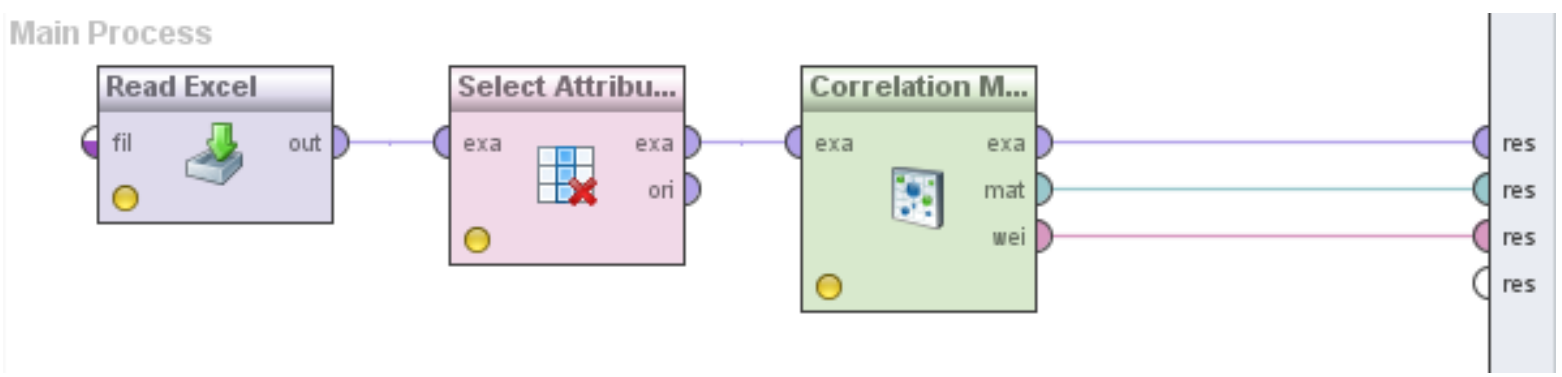


Figure 7

Correlation of Hypothetical Returns and Real Returns

\begin{tabular}{c|l|l|}
\hline \multicolumn{1}{|c|}{ Attributes } & Estimated ... & Real Return \\
\hline Estimated R & 1 & 0.192 \\
\hline Real Return & 0.192 & 1 \\
\hline
\end{tabular}




\section{Figure 8}

Data loss from November 2009 to January 2010




Figure 9

High fluctuation in the early periods of real return

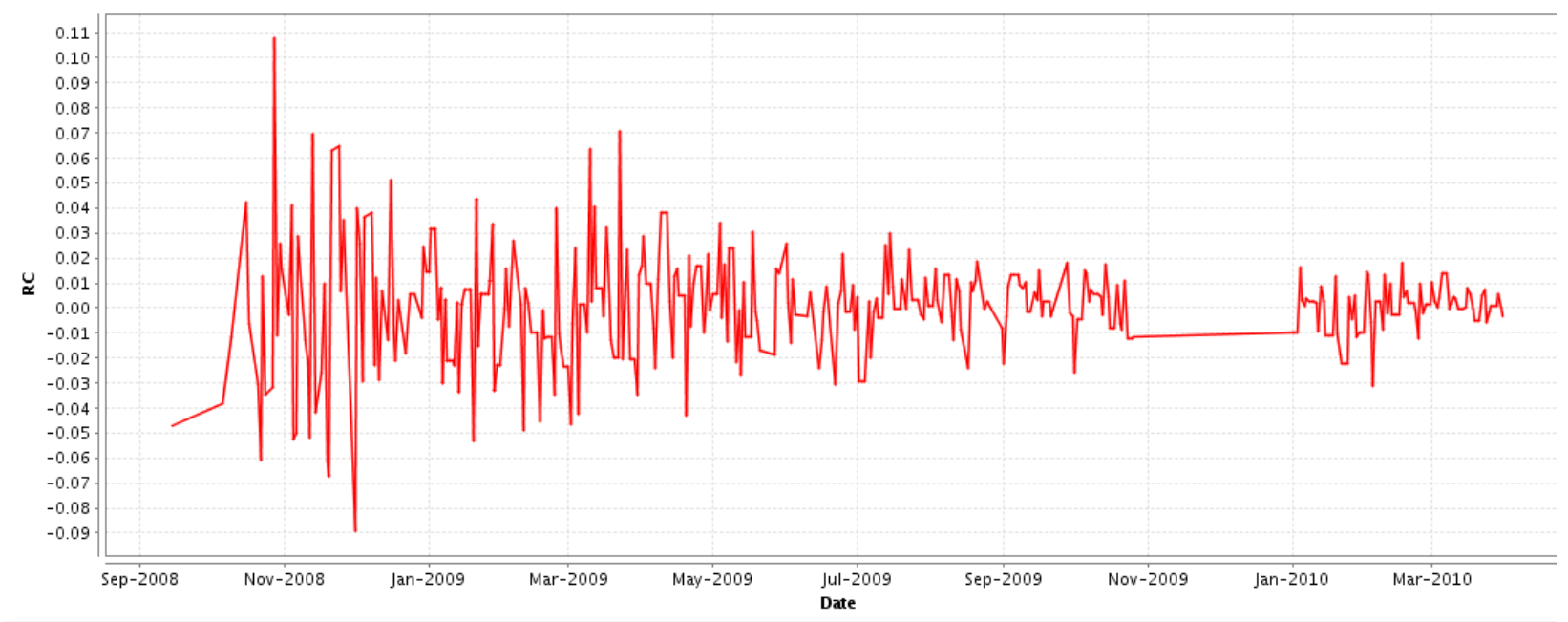


Figure 10

No visible high fluctuations in the early periods of estimated return

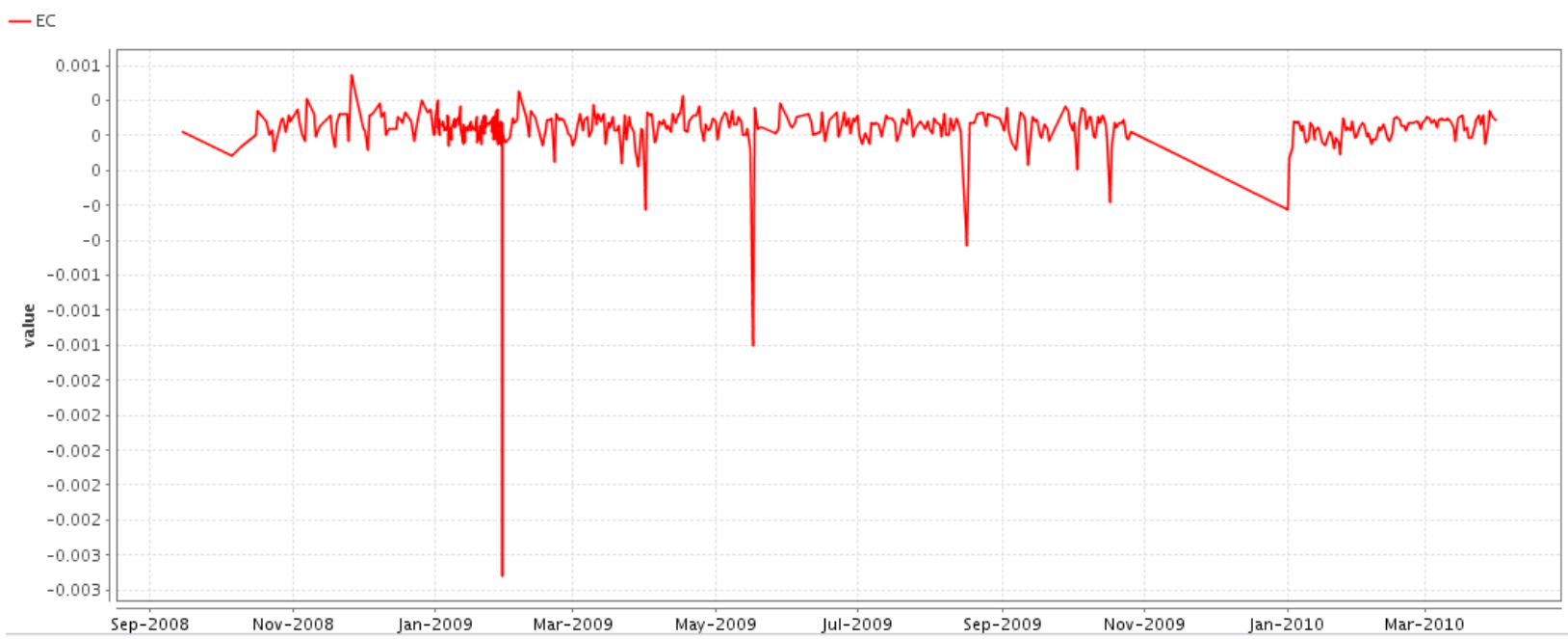




\section{Figure 11}

\section{Words Association}

\begin{tabular}{|l|l|l|}
\hline 2 & acquiring & 0.00319691 \\
\hline 3 & rally & 0.00312329 \\
\hline 4 & acquired & 0.00267631 \\
\hline 5 & breakout & 0.00266934 \\
\hline 6 & acquires & 0.00255162 \\
\hline 7 & lawsuit & 0.00245513 \\
\hline 8 & merge & 0.00232189 \\
\hline 9 & merger & 0.00231289 \\
\hline 10 & higher & 0.00229594 \\
\hline 11 & curve & 0.00227158 \\
\hline 12 & climb & 0.00215034 \\
\hline 13 & upside & 0.00205971 \\
\hline 14 & squeeze & 0.00185646 \\
\hline 15 & volume & 0.00180592 \\
\hline 16 & beta & 0.00168895 \\
\hline 17 & peak & 0.00154205 \\
\hline 18 & announcement & 0.00137009 \\
\hline 19 & past & 0.00133629 \\
\hline 20 & strike & 0.00133084 \\
\hline 21 & acquire & 0.00131280 \\
\hline 22 & monthly & 0.00129978 \\
\hline 23 & sold & 0.00127177 \\
\hline 24 & high & 0.00126894 \\
\hline 25 & chart & 0.00121377 \\
\hline 26 & gold & 0.00115793 \\
\hline 27 & gain & 0.00114301 \\
\hline & \\
\hline
\end{tabular}

(a) Words associated with positive returns

\begin{tabular}{|c|c|c|}
\hline 4 & A & B \\
\hline 117 & hate & -0.00008634 \\
\hline 118 & quarter & -0.00009115 \\
\hline 119 & loss & -0.00016146 \\
\hline 120 & debt & -0.00018902 \\
\hline 121 & euro & -0.00022953 \\
\hline 122 & china & -0.00023409 \\
\hline 123 & low & -0.00028172 \\
\hline 124 & bottom & -0.00037005 \\
\hline 125 & europe & -0.00040377 \\
\hline 126 & obama & -0.00040459 \\
\hline 127 & today & -0.00045693 \\
\hline 128 & vix & -0.00048354 \\
\hline 129 & announces & -0.00049764 \\
\hline 130 & bounce & -0.00052624 \\
\hline 131 & support & -0.00059819 \\
\hline 132 & usd & -0.00062090 \\
\hline 133 & announcing & -0.00064544 \\
\hline 134 & finance & -0.00069301 \\
\hline 135 & pound & -0.00070164 \\
\hline 136 & holdings & -0.00070749 \\
\hline 137 & down & -0.00080725 \\
\hline 138 & eps & -0.00086547 \\
\hline 139 & alpha & -0.00097745 \\
\hline 140 & futures & -0.00100139 \\
\hline 141 & year & -0.00120393 \\
\hline 142 & delta & -0.00145344 \\
\hline 143 & downside & -0.00165847 \\
\hline
\end{tabular}

(b) Words associated with negative returns 